\title{
An Optimal Strategy for Sellers in an Online Auction
}

\author{
XIN GUO \\ IBM T. J. Watson Research Center
}

We consider an online auction setting where the seller attempts to sell an item. Bids arrive over
time and the seller has to make an instant decision to either accept this bid and close the auction
or reject it and move on to the next bid, with the hope of higher gains. What should be the seller's
strategy to maximize gains? Using techniques from convex analysis, we provide an explicit closed-
form optimal solution (and hence a simple optimum online algorithm) for the seller.
Our methodology is attractive to online auction systems that have to make an instant decision,
especially when it is not humanly possible to evaluate each bid individually, when the number of
bids is large or unknown ahead of time, and when the bidders are unwilling to wait.

Categories and Subject Descriptors: F.1.2 [Computation by Abstract Devices]: Modes of Computation; G.1.6 [Numerical Analysis]: Optimization; G.1.7 [Numerical Analysis]: Ordinary Differential Equations; K.4.4 [Computers and Society]: Electronic Commerce

General Terms: Algorithms, Theory

Additional Key Words and Phrases: Convex analysis, online auctions, random walks

\section{INTRODUCTION}

An auction is a common market method to sell items. In a conventional market, the buyer considers the price of an item as given; auctions come handy in cases when the item has no predefined market value. There are many different forms of auctions, and the most common takes place in an auction house where a seller seeks the highest possible price for an item among several potential buyers. Traditionally, the buyers in an auction are called bidders and the price they seek for the item is called a bid. The birth of auctions can be dated back at least as early as $193 \mathrm{AD}$, when the Roman empire was auctioned to the highest bidder. A large volume of literature exists on auctions and auction analysis (see [Mas-Collel et al. 1995; Osborne and Rubinstein 1994]).

Author's address: IBM T. J. Watson Research Center, Yorktown Heights, NY 10598; email: xinguo@us.ibm.com.

Permission to make digital or hard copies of part or all of this work for personal or classroom use is granted without fee provided that copies are not made or distributed for profit or direct commercial advantage, and that copies show this notice on the first page or initial screen of a display along with the full citation. Copyrights for components of this work owned by others than ACM must be honored. Abstracting with credit is permitted. To copy otherwise, to republish, to post on servers, to redistribute to lists, or to use any component of this work in other works, requires prior specific permission and/or a fee. Permissions may be requested from Publications Dept, ACM Inc., 1515 Broadway, New York, NY 10036 USA, fax +1 (212) 869-0481, or permissions@acm. org.

(C) 2002 ACM 1533-5399/02/0200-0001 $\$ 5.00$ 
The growth of computers and internet technology has increased the popularity of various forms of auctions. Auctions can expose the supply-demand balances to the current dynamics of the market without significant overhead. Thanks to the internet, this overhead can be made small even for a low-value item. The internet's low-cost infrastructure enables market-makers (in this case, auction houses) to operate at a small fraction of the cost of a transaction's value. Auctions are popular on the internet via auction sites like eBay (www.ebay.com). Techniques and methodologies from auction theory have been applied to computational resource allocation and electronic commerce applications. These new settings not only provide possibilities for using various forms of auctions, but also pose interesting theoretical and algorithmic problems that arise in these contexts.

In this article, we consider one particular form of an auction. To motivate the setting, we start with an example and later abstract the problem. Our example is the allocation of communication network bandwidth by a network manager: the limited bandwidth (item) is to be sold by the network manager (seller) to different individual users or retailers (bidders). In such a situation, the bidders arrive at different times and state the price they are willing to pay for the bandwidth (bid). Upon receiving the bid, the network manager has to make an instant decision-to accept or not to accept the bid. The decision needs to be made as soon as possible from both seller and bidder points of view: waiting for more bids to arrive (if at all) will result in bandwidth underutilization and revenue loss for the seller; even worse, the bidders may be unwilling to wait for the seller's decision. This form of auction is generally called an online auction, and is studied in Lavi and Nisan [2001]. Notice that this setting is different from a traditional auction where the seller (and the buyer as well) is presumably willing to wait long enough to gather many (if not all the) bids. Also note that the network manager would have some (private) self-imposed limit on how low it is willing to sell the bandwidth.

We now state our model in generic terms. Consider a seller who has an item and sets the lowest acceptable price $l$ for the item without revealing it. Bidders arrive at different times with their bids. If a bid is lower than $l$, the seller rejects the bid. If a bid is higher than $l$, then the seller faces the decision of either accepting this bid or rejecting it and moving on to the next bidder, with the hope of higher gains. In the latter case, we assume that the rejected bid disappears. Assuming that the seller is allowed to make a choice at any time, the goal is to maximize the seller's expected return by choosing the best bid. We model this as an optimal stopping problem, and obtain an explicit solution that yields a simple algorithm for the seller.

The rest of the article is organized as follows. Section 2 presents two mathematical formulations of the auction problem, and Section 3 details the closedform solutions to these problems. Section 4 describes the online auction algorithms that are based on these solutions. Section 5 concludes the article. The Appendix contains some basic mathematical definitions used in the article and the proof of the main theorem. 


\section{MATHEMATICAL FORMULATION}

\subsection{Basic Random Walk Model}

We assume that bids arrive at discrete time steps $t=0,1, \ldots$ Let $X_{t}\left(X_{t}>0\right)$ denote the bid at time $t$ and let $M_{t}=\log X_{t}$.

We assume that the bids arrive independently, so that the differences $M_{t}-M_{s}(s \leq t)$ are independent of $\left(M_{0}, \ldots, M_{s}\right)$. Moreover, $M_{t}-M_{s}$ and $M_{t-s}-M_{0}$ are presumed to have the same distribution, i.e., $W_{t}$ has stationary increments (see Remark 2).

Furthermore, if the observation is hypothesized to be continuous, such that $M_{t}$ as a continuous process, then $M_{t}$ is in fact a Brownian motion (with drift). Namely, $M_{t}-M_{s}$ is the normal distribution with mean $\theta(t-s)$ and variance $\sigma^{2}(t-s)$ for some constants $\theta$ and $\sigma$. (See Karatzas and Shreve [1991] for background in Brownian motion and related references.)

Now let $W_{t}=X_{t+1} / X_{t}$ be the "ratio" of the bid at time $t+1$ to the bid at time $t$, so that

$$
X_{t}=X_{0} \cdot \prod_{i=1}^{t-1} W_{i}
$$

By our hypotheses, $W_{1}, W_{2}, \ldots$ are independent and identically distributed positive random variables. After each time unit, the bid price can go either up or down with certain probabilities, which can be defined as

$$
W_{t}=\frac{X_{t+1}}{X_{t}}= \begin{cases}u & \text { with probability } p, \\ d & \text { with probability } 1-p,\end{cases}
$$

where $d<1<u, 0<p<1$ are some constants.

In other words, $\left(X_{t}\right)_{t \geq 0}$ follows a geometric random walk. $X_{t}$ is nonnegative and exhibits an oscillatory behavior comprised of exponential growth intermittent with exponential decay in the long run. Moreover, for $t_{0} \leq t_{1}<\cdots<t_{n}$, the successive ratios

$$
\frac{X_{t_{1}}}{X_{t_{0}}}, \frac{X_{t_{2}}}{X_{t_{1}}}, \ldots, \frac{X_{t_{i+1}}}{X_{t_{i}}}
$$

are independent random variables, therefore the percentage change over nonoverlapping intervals are independent as well.

Let $\Delta t$ be a small time interval. If we choose $u=1 / d=\exp (\sigma \Delta t)$ and $p=\frac{e^{u \Delta t}-d}{u-d}$, then as time evolves, the well-known central limit theorem asserts that $\log \left(X_{t} / X_{0}\right)$ is a normal distribution $N\left(\theta t, \sigma^{2} t\right)$ with mean $\theta t$ and variance $\sigma^{2} t$, where $\theta=\mu-(1 / 2) \sigma^{2}$. (See Karatzas and Shreve [1991], for instance.)

In other words, we can write $M_{t}$ as

$$
\Delta M_{t}=\theta \Delta t+\sigma \eta \sqrt{\Delta t},
$$

and $X_{t}$ as

$$
\frac{\Delta X_{t}}{X_{t}}=\mu \Delta t+\sigma \eta \sqrt{\Delta t}
$$


where $\eta$ is the normal distribution $N(0,1)$ with zero mean and unit variance. Here by the central limit theorem, $\theta=\mu-(1 / 2) \sigma^{2}$.

The following are basic facts about our model (see Karatzas and Shreve [1991]).

Proposition 1.

(1) $E\left[\left(\Delta M_{t}\right)^{2}\right]=\sigma^{2} \Delta t$.

(2) $E\left[\Delta X_{t}\right]=\mu \Delta t$

(3) $\operatorname{Var}\left[\Delta X_{t}\right]=\sigma^{2} \Delta t$.

(4) When $\mu=0$ (or $\theta=0$ ), $X_{t}$ (or $M_{t}$ ) is a martingale.

Remark 1. Random walk (or Brownian motion and its like) has been widely used in mathematical finance for modeling stock-price fluctuations [2]. Despite similarities in activities between the stock trading floor and the auction house (for instance, "bid" and "ask"), approaches and perspectives in auction theory and mathematical finance have been quite different.

With the fast growth of online auctions and online stock trading and their ever faster convergence, it is worth exploring the possibility of adopting some ideas from mathematical finance and apply them to online auctions.

Remark 2. In our random walk model, we asserted the stationary assumption for $M_{t}$. However, when such a model is applied to problems such as auctions for network connections, appropriate choice of the time window is critical, because higher bids may take place around peak times, and consequently bid differences may not be uniform. Given the ever-increasing volume of online auctions, it is very difficult, and even unrealistic, to assume good knowledge of potential bids among a large number of diverse bidders who are no longer physically restricted inside an auction house. The question of what kind of distribution other than Brownian motion, if there is any, best describes online bids remains a challenging problem. Until statistical studies provide definite clues, this stationary assumption is a tradeoff for technical tractability as a first-stage approximation.

\subsection{Problem Formulation}

Now consider the random walk $X_{t}$ (hence, $M_{t}$ ), with $X_{t}$ (or $M_{t}$ ) the bid of an auction at time $t$. At each time $t$ the seller has the option of either stopping the auction and obtaining the reward given by some reward function $g\left(X_{t}\right)$, or continuing the auction in the hope that stopping it at a later time will garner a bigger reward (minus some transaction costs). Of course, if there is no loss of revenue in waiting and if the mean of the process is positive, the seller may choose to continue the auction until he is satisfied. However, if there is a transaction cost and the best bid may disappear after a certain time delay and there is an uncertainty in the state of the auction at any future time, then the seller may not continue the auction forever, and has to stop at some point. For any $t$, the decision to stop at any $\tau<t$ depends only on the behavior of $X_{u}$ for $u \leq t$. Among all these stopping times, the seller looks for the optimal time $\tau^{*}$ which gives the best result in the long run. Since bids are assumed to be stochastic, the seller is effectively looking for the best expected value of the bid. 
If we assume the reward function $g$ is continuous and nonnegative, then the question is to find a stopping time $\tau^{*}$ for $\left\{X_{t}\right\}$ (or $M_{t}$ ) such that

$$
E_{x}\left[g\left(X_{\tau^{*}}\right)\right]=\sup _{\tau} E_{x}\left[g\left(X_{\tau}\right)\right]
$$

for all $x$ where sup takes all stopping time $\tau$ for $\left\{X_{t}\right\}$. The corresponding optimal expected reward is

$$
g^{*}(x)=E_{x}\left[g\left(X_{\tau^{*}}\right)\right] .
$$

Here $E_{x}$ denotes the expectation with respect to the law of probability for the process $X_{t}, t \geq 0$ starting at $X_{0}=x$.

Our online auction setting is captured precisely by the above formulation, except for two issues. First is that, in the auction setting, the seller has a (private) lowest price $l$ for the auction. And second is that the reward function is as yet unspecified. Different sellers can choose different reward functions to optimize. Incorporating these issues, and based on the mathematical model proposed in Section 2.1, we consider the following two optimization problems with different reward functions. The first reward function is linear, while the second is exponential.

Problem A. In Eq. (2), let $M_{0}=m$ and let $H_{t}=\max \left(M_{t}, l\right)$. Suppose the transaction cost $r>0$ is proportional to the time the auction lasts, and the reward function is

$$
g\left(M_{t}\right)=H_{t}-r t
$$

then what is the value of

$$
V^{*}(m, l)=\sup _{0 \leq \tau \leq \infty} E_{m, l}\left[H_{\tau}-r \tau\right] ?
$$

Problem B. In Eq. (3), let $X_{0}=x$ and $Y_{t}=\max \left(X_{t}, l\right)$. Suppose there is a "discounted factor" $r>0$ (for example, 1 dollar today is equivalent to 1.5 dollars in one month), and the reward function is

$$
g\left(X_{t}\right)=e^{-r t} \max \left\{X_{t}, l\right\}=e^{-r t} Y_{t},
$$

then what is the value of

$$
H^{*}(x, l)=\sup _{0 \leq \tau \leq \infty} E_{x, l}\left[e^{-r \tau} Y_{\tau}\right] ?
$$

We may have realized that these two problems are mathematically related, just as the arithmetic mean and the geometric mean. Therefore, some similarity in the structure of the solutions is anticipated. Indeed as our analysis goes, it shows that the optimal stopping strategy is based on the difference $x-l$ for the first case and on the ratio $x / l$ for the second one-although the optimal value functions are rather different.

Remark 3. Some skepticism is expected about the formulation of the problems using "discounted factors." In contrast to financial markets where investments might take up to several years, many auctions may last less than a few 
days, and the effect of "inflation" (money devaluation) is much less obvious. So what are the "discounted factors"? The transaction cost is one candidate. Another less explicit yet important candidate is the uncertainty embedded in any future contract. A short time span, however brief it might be, carries risks and generates value differences. To exemplify this, consider an online bidding for an airline ticket: from a seller's point of view, a 300 dollar bid for a ticket three weeks before the flight is not the same as a 300 dollar bid the day before the flight. As an another example, consider online auctions for flowers: the value of the item is quickly lost over time, and this can be captured by the "discounted factor." Hence, we have to interpret the "discounted factor" in a broader sense than that of the "interest rate" factor of the financial industry.

We remark that our analysis actually applies to any constant $r$. Therefore, the interpretation of $r$ as a discounted factor is for ease of exposition.

\section{SOLUTIONS}

\subsection{Problem A}

In the following, we first give an intuitive guess at the right solution and then prove it rigorously.

Let $t=0$, with $M_{0}=m, H_{0}=\max (m, l)$, where $m$ is the current bid. Intuitively, if $m$ is already very high (no less than $l$ ), then chances that the seller will get a better bid later is not high because the transaction cost might take away all the increment. So, perhaps we should stop and receive the reward $m$. On the other hand, if $m$ is too low, for example when $m<l$, then we may need to wait for quite long time in order to get a bid that is at least $l$, and the transaction cost will offset any big return. So, again, chances that we will get a bid better than $l$ is low. Therefore, wisdom suggests that we stop and collect the reward $l$. This intuition directs us to seek a (smooth) value function $V(m, l)$ and some functions $a(l)$ and $b(l)$, such that when $a(l)<m<b(l)$, we should continue getting the bid. Here we call the region $a(l)<m<b(l)$ the continuation region. When $0 \leq m \leq a(l)$, or $m \geq b(l)$, we should stop. We call this the stopping region. The boundary between the continuation region and the stopping region is generally referred to as the "free boundary." In many optimal stopping problems, the "free boundary" uniquely determines the value function.

Our intuition for the guess of the "free boundary" can be translated mathematically, as follows: Suppose our best value function $V(m, l)$ is continuous and smooth. For any fixed $l$ at time $t=0$, if we decide to continue for a small time interval $h>0$, we have

$$
V(m, l) \geq E[V(m+\theta h+\sigma \sqrt{h} \eta, l)-r h],
$$

Since $V(m, l)$ is the best we can achieve. Expanding this using Taylor series plus Proposition 1, we get

$$
V(m, l) \geq V(m, l)+\theta h V_{m}+(1 / 2) x^{2} \sigma^{2} h V_{m m}-r h .
$$

which is

$$
\theta V_{m}+(1 / 2) \sigma^{2} V_{m m}-r \leq 0 .
$$

ACM Transactions on Internet Technology, Vol. 2, No. 1, February 2002. 
Apparently, when we stop and get the maximal value, the inequality should be replaced by "=". It is not difficult to see that $V(m, l) \geq \max (m, l)$, since we can always choose to stop immediately. Moreover, $V(m, l)$ is increasing with respect to $m$. If we also expect the value function to be smooth along the "free boundary," then we "guess" that Problem A can be solved by finding the solution to the following alternative problem (also called "variational inequality"):

Problem A1. Find $a$ and $b$ such that $0 \leq a<l<b \leq \infty$, and an increasing convex function $V(\cdot, l)$ in the space $C^{2}(a, b) \cap C^{1}[a, b] \cap C^{2}(-\infty, a) \cap C^{2}(b, \infty)$ such that

$$
\begin{aligned}
\theta V_{m}+(1 / 2) \sigma^{2} V_{m m}-r & =0, \quad m \in(a, b), \\
\theta V_{m}+(1 / 2) \sigma^{2} V_{m m}-r & \leq 0, \quad m \notin(a, b), \\
V(m, l) & \geq l, m \\
V(a, l) & =l, \\
V(m, l) & =m, \quad m \geq b .
\end{aligned}
$$

Proposition 2. The solution to Problem $A 1$ is given by $V(m, l)$ where:

(1) when $r=0>\theta, V(m, l) \equiv l$;

(2) when $r=\theta>0$,

$$
V(m, l)= \begin{cases}l, & m<l-\frac{\sigma^{2}}{2 r}, \\ m+\frac{\sigma^{2}}{2 r} \exp \left(-\frac{2 r}{\sigma^{2}}\left(m-l+\frac{\sigma^{2}}{2 r}\right)\right), & m \geq l-\frac{\sigma^{2}}{2 r} ;\end{cases}
$$

(3) when $r>\max (0, \theta)$,

$$
V(m, l)= \begin{cases}l, & m \leq a, \\ l+\frac{r}{\theta}(m-a)-\frac{r \sigma^{2}}{2 \theta^{2}}\left(1-\exp \left(-\frac{2 \theta}{\sigma^{2}}(m-a)\right)\right), & a<m<b, \\ m, & b \leq m,\end{cases}
$$

with $l>a=l+\frac{\sigma^{2}}{2 \theta}\left(\frac{r-\theta}{\theta} \ln \frac{r-\theta}{r}-1\right), l<b=l+\frac{\sigma^{2}}{2 \theta}\left(\frac{r}{\theta} \ln \frac{r}{r-\theta}-1\right)$.

Proof. Using $e^{-x} \geq 1-x$, we see $V(m, l) \geq \max (m, l)$. To see that $l<b$ when $r>\theta>0$, it suffices to show that $\frac{r}{\theta} \ln \frac{r}{r-\theta}>1$. This is true because

$$
\ln \frac{r}{r-\theta}=\ln \left(1+\frac{\theta}{r-\theta}\right) \geq \frac{\theta}{r-\theta}>\frac{\theta}{r} .
$$

The rest can be proved in a similar way by simple calculations.

The following theorem (with its martingale proof in Appendix B) shows that the solution we argued heuristically is in fact optimal.

Theorem 3. (1) When $r<\max (0, \theta), P(\tau<\infty)=0$, and $V^{*}(m, l)=\infty$; (2) when $r \geq \max (0, \theta), V(\cdot, l)$ is the optimal expected reward of the stopping Problem $A 1$, i.e., $V^{*}(m, l)=V(m, l)$ and the optimal stopping time is

$$
\tau_{*}=\inf \left\{t \geq 0 \mid X_{t} \notin(a, b)\right\} .
$$


(We interpret the case $r=0>\theta$ as $a=0, b=\infty$, and the case $r=\theta>0$ as $b=\infty, a=l-\frac{\sigma^{2}}{2 r}$.)

\subsection{Problem B}

As before, our first step is to heuristically "guess" a solution to Problem B. Since the underlying process in this case is a geometric Brownian motion, we guess that the optimal stopping rule should be based on $x / l$ (instead of $x-l$ ). Again, as before, it is not hard to see that solving Problem B is equivalent to solving the following problem:

Problem B1. Find two numbers $0 \leq a \leq 1<b \leq \infty$ and an increasing $g(x)=g(\cdot, l)$ in the space $C^{2}(a l, b l) \cap C^{1}[a l, b l] \cap C^{2}(0, a l) \cap C^{2}(b l, \infty)$, such that

$$
\begin{aligned}
x \mu g_{x}+1 / 2 x^{2} \sigma^{2} g_{x x}-r g & =0, \quad x \in(a l, b l), \\
x \mu g_{x}+1 / 2 x^{2} \sigma^{2} g_{x x}-r g & \leq 0, \quad x \notin(a l, b l), \\
g(x, l) & \geq l, \quad x \\
g(a, l) & =l, \\
g(x, l) & =x, \quad x \geq b l .
\end{aligned}
$$

In order to solve Problem B1, we define $0<a<1<b$ such that when $r>\max (0, \mu)$,

$$
b=\frac{\gamma_{0}}{\gamma_{0}-1}\left(\frac{\gamma_{0}\left(\gamma_{1}-1\right)}{\gamma_{1}\left(\gamma_{0}-1\right)}\right)^{\frac{\gamma_{1}}{\gamma_{0}-\gamma_{1}}}
$$

and

$$
a=\frac{\gamma_{1}}{\gamma_{1}-1}\left(\frac{\gamma_{1}\left(\gamma_{0}-1\right)}{\gamma_{0}\left(\gamma_{1}-1\right)}\right)^{\frac{1-\gamma_{0}}{\gamma_{0}-\gamma_{1}}},
$$

with $\gamma_{0}>1>0>\gamma_{1}$ satisfying $r=\mu \gamma+1 / 2 \sigma^{2} \gamma(\gamma-1)$.

Proposition 4. Solution to Problem B1 is given by the following function $g(x, l)$, such that

(1) when $r>\max (0, \mu)$,

$$
g(x, l)=\left\{\begin{array}{lr}
l & x \leq a l<l, \\
l\left(\left(\frac{x}{a l}\right)^{\gamma_{1}}\left(\frac{\gamma_{0}}{\gamma_{0}-\gamma_{1}}\right)-\left(\frac{x}{a l}\right)^{\gamma_{0}}\left(\frac{\gamma_{1}}{\gamma_{0}-\gamma_{1}}\right)\right) & a l<x<b l, \\
x & l<b l \leq x ;
\end{array}\right.
$$

where $a, b$ as defined by Eq. (19), and (20);

(2) when $r=\mu>0$,

$$
g(x, l)= \begin{cases}l & x \leq l, \\ l\left(\left(\frac{x}{l}\right)^{\gamma_{1}}\left(\frac{\gamma_{0}}{\gamma_{0}-\gamma_{1}}\right)-\left(\frac{x}{l}\right)^{\gamma_{0}}\left(\frac{\gamma_{1}}{\gamma_{0}-\gamma_{1}}\right)\right) & l<x ;\end{cases}
$$

(3) when $r=0>\mu, g(x, l) \equiv l$. 
Theorem 5. (1) When $r<\max (0, \mu), P(\tau<\infty)=0$, and $H^{*}(x, l)=\infty$;

(2) when $r \geq \max (0, \mu), g(\cdot, l)$ is the optimal expected reward of the stopping Problem B1, i.e., $H^{*}(x, l)=g(x, l)$, and the optimal stopping time is

$$
\tau_{*}=\inf \left\{t \geq 0 \mid X_{t} \notin(a l, b l)\right\} .
$$

(Here we interpret the case $r=0>\mu$ as $a=0, b=\infty$, and the case $r=\mu>0$ as $b=\infty, a=1$.)

Remark 4. The methodology we present in this article can be easily applied to a different auction type where the seller has a highest level in mind and tries to minimize the total cost. The mathematical formulation is dual to Problems $\mathrm{A}$ and $\mathrm{B}$, i.e.,

$$
G^{*}(x, l)=\inf _{\tau} E\left[g\left(X_{\tau}\right)\right],
$$

where the inf is taken over all stopping times $\tau$, and $g$ is the utility function.

\section{MEMORYLESS ALGORITHMS FOR ONLINE SELLERS}

Since our theorems produce explicit solutions, they yield straightforward algorithms. For the algorithms, we assume that bids are lost if not immediately accepted. For convenience, we assume $r>0$. Notice that in Problem A, when $r=\theta>0$, for those who seek bids better than $l$ will only get an almost optimal strategy, and not the optimal strategy, since $b \rightarrow \infty$.

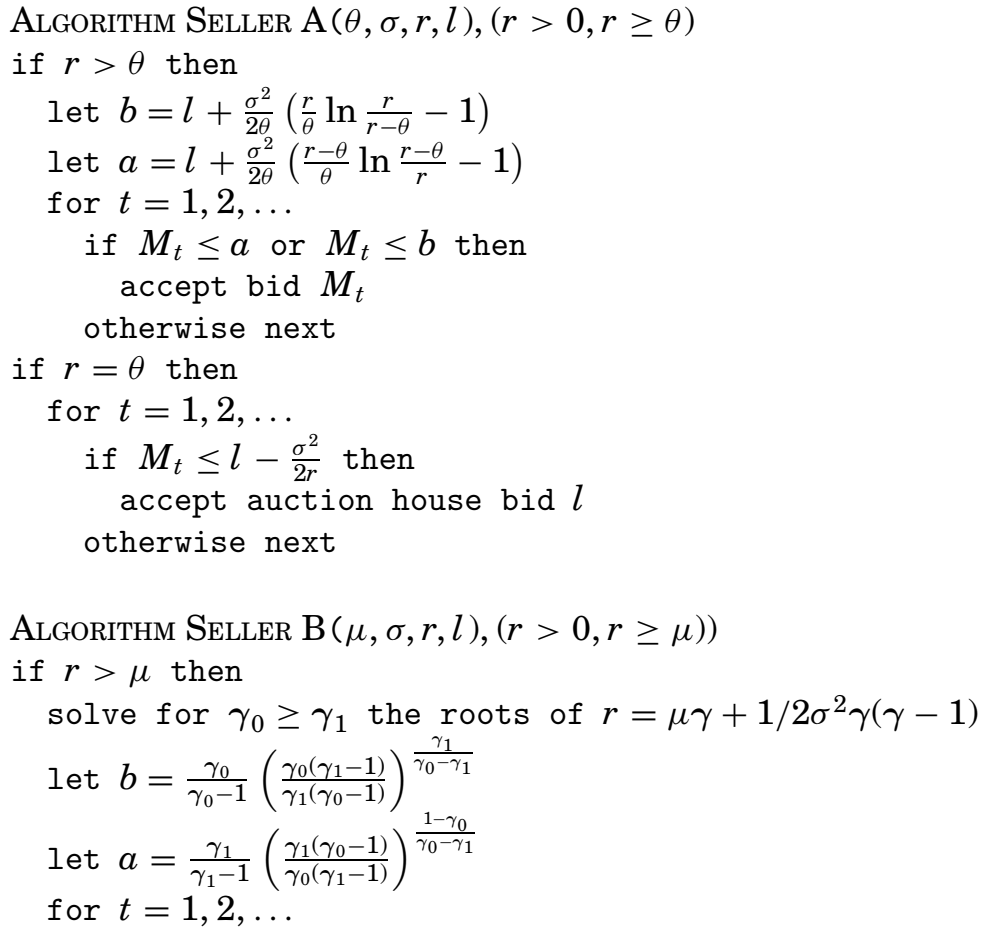




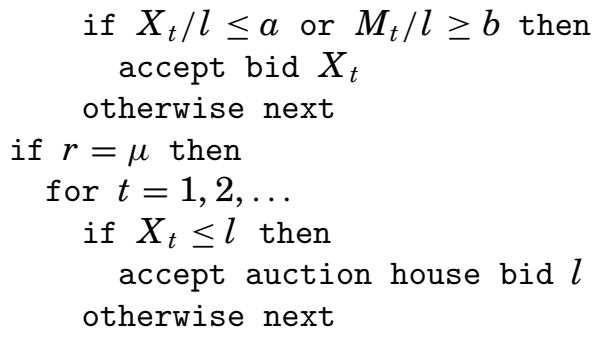

It is worth pointing out that if there are no transaction costs or discounted factors, i.e., when $r<\max (0, \mu)$ (or $r<\max (0, \theta)$ ), the optimal strategy for Problem B (or Problem A) is simple: wait!

\section{CONCLUDING REMARKS}

In this article we presented a random walk model for the bids of an online auction and provided explicit solutions to some optimization problems for sellers.

The advantages of presenting explicit solutions to problems for a continuous case is that it provides insight into the solution structure and leads to efficient algorithms. This is especially appealing for online decisions, when it is not humanly possible to evaluate each bid individually, when the number of bids is large or unknown ahead of time, and when the bidders are unwilling to wait. Furthermore, in cases where no optimal strategy is available, as in our case when $r=\mu$ or $r=\theta$, and we argued that only almost optimal strategies exist, our analysis provides useful information and guidance for offline and/or discrete algorithms. Finally, with the closed-form solution, our algorithm is memoryless-it does not store previous bids and makes decision based solely on the current bid.

We acknowledge that our auction setting can be argued as slightly unrealistic because of the indefinite time horizon. In practice, bidders' behavior would be very different if there were a definite deadline (for example, some bidders might wait until the last minute). But it is hard to solve the corresponding finite time horizon optimization problem with a closed-form solution. Hence, assuming an infinite time horizon gives us a reasonably good asymptotic approximation to the otherwise intrinsically complicated auctions, while retaining the tractability and simplicity of the solution. We also hope our analysis can be useful in combinatorial auctions to reduce computational complexity. As is well known, in combinatorial auctions, more than one item is bundled to sell, and the computational difficulty increases exponentially.

Moreover, even though it is hard to get a closed-form solution for the finite horizon case, it is possible to get a numerical result based on our analysis. It will be interesting to see whether there is a polynomial time approximation to our problems in the finite time horizon case and in the case where distributions other than the random walk model are assumed.

Finally, we note that if we allow bidders with rejected bids to come back (usually with higher bids), then there might be some bias in the distribution of the bid price, and we should be cautious about the assumption of independent increment. This case is worth further research. 
APPENDIX A. OPTIMAL STOPPING PROBLEMS

Definition 6. Let $(\Omega, \mathcal{F}, P)$ be a probability space, let $\left\{N_{t}\right\}$ be an increasing family of $\sigma$ - algebras (subset of $\Omega$ ). A function $\tau: \Omega \rightarrow[0, \infty]$ is called a stopping time with respect to $N_{t}$ if

$$
\{\omega: \tau(\omega) \leq t\} \in N_{t},
$$

for all $t \geq 0$. In other words, whether or not $\tau \leq t$ has occurred is based completely on the knowledge of $N_{t}$.

If we refer to the sequence of random variables $\left(\left\{X_{t}\right\},\left\{M_{t}\right\}\right)$ as a process, then

Definition 7. A process $\left\{X_{t}\right\}$ is adapted iff $X_{t}$ is $N_{t}$ measurable.

Definition 8. An adapted process $X_{t}$ is a martingale iff for any $s \leq t$,

$$
E\left[X_{t} \mid N_{s}\right]=X_{s} .
$$

The process is said to be a super(sub) martingale if the above relation holds with $\leq(\geq)$ replacing the equality.

Definition 9. An adapted process $X_{t}$ is a local martingale iff for any $S \leq T$, and any stopping time $\tau$,

$$
E\left[X_{\min (T, \tau)} \mid N_{S}\right]=X_{S}
$$

A martingale is naturally a local martingale, while the converse is not necessarily true.

Theorem 10. (Optional sampling) [Karatzas and Shreve 1991]. Let $\left(X_{t}, N_{t}, 0 \leq t<\infty\right)$ be a right-continuous submartingale with a last element $X_{\infty}$, and let $S \leq T$ be two optional time of the filtration $\left\{N_{t}\right\}$. Almost surely we have,

$$
E\left[X_{T} \mid N_{t}\right] \geq X_{S}
$$

In particular, $E X_{T} \geq E X_{0}$, and for a martingale with a last element, we have $E X_{T}=E X_{0}$.

APPENDIX B. PROOF OF THEOREM 3

Define $Z_{t}=V\left(M_{t}, l\right)-r t$. Then $Z_{t}$ is expectation-decreasing $\left(Z_{t}\right.$ is a positive supermartingale),

$$
E_{x, l}\left[d Z_{t}\right] \leq 0 .
$$

This is due to Karatzas and Shreve [1991] and Klemperer [1999], and

$$
\left.E\left[d Z_{t}\right]=\left[-r+\theta V_{m}^{\prime}+1 / 2 \sigma^{2} V_{m m}^{\prime \prime}\right)\right] d t \leq 0 .
$$

Recall that $V(m, l) \geq \max (m, l)$, therefore, we have, for any stopping time $\tau$,

$$
E_{m, l}\left[M_{\tau}-r \tau\right] \leq E_{m, l}\left[V\left(X_{\tau}, l\right)-r \tau\right]=E_{m, l}\left[Z_{\tau}\right] \leq E_{m, l}\left[Z_{0}\right]=V(m, l) .
$$

Hence, taking the supremum over all stopping times,

$$
\sup _{\tau} E_{m, l}\left[M_{\tau}-r \tau\right] \leq V(m, l)
$$


and

$$
V^{*}(m, l) \leq V(m, l) .
$$

Moreover, from Shepp and Shiryaev [1993] we know that $E \sup _{t} Z_{t}<\infty$. Therefore, $Z_{t}$ is a martingale when $M_{t} \in(a, b)$, and we can apply the optional stopping theorem such that for any stopping time $\tau$,

$$
E_{m, l}\left[Z_{\tau}\right]=E_{m, l}\left[Z_{0}\right] .
$$

Taking the supremum over all stopping times, we have

$$
V^{*}(m, l) \geq V(m, l) .
$$

Therefore,

$$
V^{*}(m, l)=V(m, l)
$$

\section{ACKNOWLEDGMENTS}

The author would like to thank Michael Rothkopf, for comments on an early draft, and the anonymous referee for various suggestions in improving the presentation.

\section{REFERENCES}

Aminud, Y. ed. 1976. Bidding and Auctioning for Procurement and Allocation. New York University Press.

Black, F. and Scholes, M. 1973. The pricing of options and corporate liabilities. J. Political Econ. 81, 637-654.

Capen, E., Clapp, R., and Campbell, W. 1971. Competitive bidding in high risk situation. J. Petroleum Technol. 23, 641-653.

Chernoff, H. 1961. Sequential tests for the mean of a normal distribution. In Proceedings of the 4th Berkeley Symposium Stat. Prob. 1: 79-91.

Engelbrecht-Wiggans, R., Shubik, M., and Stark, R. M. eds. 1983. Auctions, Bidding, and Contracting: Use and Theory. New York University Press.

Friedman, L. 1956. A Competitive bidding strategy. Oper. Res. 4, 104-112.

Guo, X. 2001. An explicit solution to an optimal stopping problem with regime switching. J. Appl. Probab. 38, 2.

Karatzas, I. And Shreve, S. 1991. Brownian Motion and Stochastic Calculus. Springer Verlag.

Klemperer, P. 1999. Auction theory: A guide to the literature. J. Econ. Surv. 13, 3, 227-286.

Lavi, R. AND Nisan, N. 2001. Competitive analysis of incentive compatible on-line auctions, Preprint.

Mas-Collel, A., Whinston, W., And Green, J. 1995. Microeconomic Theory, Oxford University Press.

Milgrom, P. R. AND Weber, R. J. 1982. A theory of auctions and competitive bidding. Econometrica, $50,1089-1122$.

Myerson, R. B. 1981. Optimal auction design. Math. Oper. Res. 6, 58-73.

McAfee, R. P. and McMillan, J. 1987. Auctions and biddings. J. Econ. Lit. 25, 699-738.

Oren, M. E. ANd William, A. C. 1975. On competitive bidding. Oper. Res. 23, 1072-1079.

Osborne, M. J. And Rubinstein, A. 1994. A Course in Game Theory, MIT Press, Cambridge, MA.

Riley, J. G. And SAmuelson, W. 1981. Optimal auctions. Am. Econ. Rev. 71, 381-392.

Robbins, H., Sigmund, D., And Chow, Y. 1971. Great Expectations: The Theory of Optimal Stopping, Houghton-Mifflin.

RothKopf, M. H. AND HARSTAD, R. M. 1994. Modeling competitive bidding: a critical essay. Manage. Sci. $40,3,364-384$.

ACM Transactions on Internet Technology, Vol. 2, No. 1, February 2002. 
Shepr, L. and Shiryaev, A. N. 1993. The Russian option: Reduced regret. Ann. Appl. Probab. 3, 631-640.

STARK, R. M. 1974. Unbalanced highway contracting. Oper. Res. Q. 25, 373-388.

Wilson, R. B. 1993. Strategic analysis of auctions. In The handbook of Game Theory, R. Aumann and S. Hart, Eds. North-Holland/Elsevier Science.

Received December 2000; revised March 2001; accepted November 2001 